\title{
Analysis and prediction of crack propagation in plates by the enriched free Galerkin method
}

\author{
Bui Manh Tuan ${ }^{1,2, *}$, Chen Yun Fei ${ }^{1}$ \\ ${ }^{1}$ School of Mechanical Engineering, Southeast University, Nanjing city, Jiangsu Province, China \\ ${ }^{2}$ Faculty of Mechanical Engineering, Tuy Hoa Industrial College, Tuy Hoa City, Phu Yen Province, Vietnam \\ Email address: \\ buimanhtuan.tic@gmail.com (B. M. Tuan),yunfeichen@seu.edu.cn (Chen Yunfei)
}

\section{To cite this article:}

Bui Manh Tuan, Chen Yun Fei. Analysis and Prediction of Crack Propagation in Plates by the Enriched Free Galerkin Method. International Journal of Mechanical Engineering and Applications. Vol. 2, No. 6, 2014, pp. 78-86. doi: 10.11648/j.ijmea.20140206.11

\begin{abstract}
This paper presents a centre and edge crack analysis using meshless methods which is based on moving least squares (MLS) approximation. The unknown displacement function $\mathrm{u}(\mathrm{x})$ is approximated by moving least square approximation $\mathrm{u}^{\mathrm{h}}(\mathrm{x})$. These approximation are constructed by using a weight function which is based a monomial basis function and a set of non-constant coefficients. A subdivision that is similar to finite element method is used to provide a background mesh for numerical integration. An enriched EFG formulation with fracture problems is proposed to improve the solution accuracy for linear elastic fracture problem. The essential boundary conditions are enforced by Lagrange multipliers method. A code has been written in Matlab for the analysis of a crack tip. The obtained results of the developed EFG-code were compared to available experimental data and other numerical (exact methods and finite element method) methods.
\end{abstract}

Keywords: Crack, Stress Intensity Factor, EFG Method, Moving Least Squares Approximant, Crack Propagation

\section{Introduction}

There are many numerical methods applied for modeling cracks in mechanical problems such as the Boundary Element Method (BEM) [1], the Finite Element Method (FEM) [2], the extended Finite Element Method (XFEM) [3] and Meshless Methods (MMs). Among these methods, the element free Galerkin method and enriched element free Galerkin method has developed by Belytschko et al. [4,5] and it has been widely applied in fracture mechanics [6-8]. The meshless method works better than the traditional finite element method (FEM) in treatment of arbitrary evolving discontinuity. Due to the elements are independent of each other so the problems of crack analysis is simplified considerably which can be analyzed on a fixed mesh and unnecessary remeshing for crack development issues.

In this paper, we use a meshless method to analyze twodimensional elastic problem by using Element-Free Galerkin (EFG) method that is based on moving least squares approximation (MLS) to construct the function approximation for the Galerkin weak form. These approximations are constructed by using a weight function that base on a monomial basis function and a set of nonconstant coefficients. A subdivision similar to finite element method is used to provide a background mesh for numerical integration. The necessary boundary conditions are implemented by means of Lagrange multipliers. The method of enriched EFG that is enriched basis and nodal refinement is utilized to calculate and simulate crack growth. Therefore, the precision calculation is improved. The continuous crack propagation is modeled as a linear series of crack growth. In there, plate for cracks in the center and edge cracks is presented in this paper. The results obtained for twodimensional problem with different of the number of nodes, crack length, load and dimensionless size of the support domain in the region of the crack tip compare to element finite method and exact method. In addition, Matlab code of EFG method also is offered in this paper. This Matlab code can be developed for meshfree application software or other meshfree method in the further.

\section{MLS Approximations Functions}

MLS functions were developed by Lancaster and Salkauskas in the literature [9] to approximate curves and surfaces, and then was used in EFGM method to generate shape functions [1,9]. In EFGM, a field variable $u(x)$ is approximated by MLS approximation, $\mathrm{u}^{\mathrm{h}}(\mathrm{x})$ [22] which is given as 


$$
u(x) \cong u^{h}(x)=p^{T}(x) \cdot a(x) \quad \forall x \in \Omega_{x}
$$

Where $\mathrm{p}(\mathrm{x})$ is a linearly independent basis of $\mathrm{m}$ functions

$$
\mathrm{p}^{\mathrm{T}}(\mathrm{x})=\left[\mathrm{p}_{1}(\mathrm{x}) \mathrm{p}_{2}(\mathrm{x}) \ldots \mathrm{p}_{\mathrm{m}}(\mathrm{x})\right]
$$

And $a(x)$ collects the undetermined parameters of the approximation

$$
a(x)=\left[a_{1}(x) a_{2}(x) \ldots a_{m}(x)\right]^{T}
$$

The $\mathrm{a}(\mathrm{x})$ parameters are obtained by minimizing a weighted least square sum. The weighted least square sum denoted by $\mathrm{L}(\mathrm{x})$ can be written as follows:

$$
\begin{aligned}
L(x) & =\sum_{I=1}^{n} \omega\left(x-x_{I}\right) \cdot\left[\mathrm{u}^{h}\left(x_{I}, \mathrm{x}\right)-u_{I}\right]^{2} \\
& =\sum_{I=1}^{n} \omega\left(x-x_{I}\right) \cdot\left[\mathrm{p}^{T}\left(x_{I}\right) a(x)-u_{I}\right]^{2}
\end{aligned}
$$

Where $\omega\left(\mathrm{x}-\mathrm{x}_{\mathrm{I}}\right)$ is a weighting function which is nonzero on the influence domain of the node $\mathrm{x}_{\mathrm{I}} ; \mathrm{n}$ is the number of points in the neighbourhood of $x$, and $u_{I}$ is the nodal value of $\mathrm{u}$ at $\mathrm{x}=\mathrm{x}_{\mathrm{I}}$. The dimension of the influence domain of each node and the choice of the weighting function are decisive parameters for the approximation by MLS [4].

Minimizing $\mathrm{L}(\mathrm{x})$ in order to the unknown parameters $\mathrm{a}(\mathrm{x})$ results in

$$
A(x) \cdot a(x)=B(x) \cdot u
$$

$\mathrm{Or}$

$$
a(x)=A^{-1}(x) \cdot \mathrm{B}(x) \cdot u
$$

With:

$$
\begin{array}{r}
A(x)=\sum_{I=1}^{n} w\left(x-x_{I}\right) \cdot p\left(x_{I}\right) \cdot p^{T}\left(x_{I}\right) \\
\mathrm{B}(x)=\left[w\left(x-x_{1}\right) \cdot p\left(x_{1}\right), w\left(x-x_{2}\right) \cdot p\left(x_{2}\right), \ldots\right. \\
\left., w\left(x-x_{n}\right) \cdot p\left(x_{n}\right)\right]
\end{array}
$$

Substituting the result (5) in the initial approximation (1), the MLS approximation is obtained as:

$$
u^{h}(x)=\sum_{I=1}^{n} \Phi_{I}(x) \cdot u_{I}=\Phi(x) U
$$

Where the shape function is defined by

$$
\Phi_{I}(x)=\sum_{j=0}^{m} p_{j}(x)\left(A^{-1}(x) B(x)\right)_{j I}=p^{T} A^{-1} B_{I}
$$

Where $\mathrm{m}$ is the order of the polynomial $\mathrm{p}(\mathrm{x})$. To determine the derivatives from the displacement (8), it is necessary to obtain the shape function derivatives. The spatial derivatives of the shape functions are obtained by:

$$
\Phi_{I, x}=\left(p^{T} A^{-1} B_{I}\right)_{, x}=p_{, x}^{T} A^{-1} B_{I}+p_{, x}^{T}\left(A^{-1}\right)_{, x}+p^{T} A^{-1} B_{I, x}
$$

Where

$$
\begin{aligned}
& B_{I, x}(x)=\frac{d \omega}{d x}\left(x-x_{I}\right) p\left(x_{I}\right) ; \\
& A_{, x}^{-1}(x)=-A^{-1} A_{, x} A^{-1} ; \\
& A_{, x}=\sum_{I=1}^{n} \omega\left(x-x_{I}\right) p\left(x_{I}\right) p^{T}\left(x_{I}\right)
\end{aligned}
$$

It should be noted that EFG shape functions do not satisfy the Kronecker delta criterion: $\Phi_{\mathrm{I}}(\mathrm{x})^{1} \neq \delta_{\mathrm{ij}}$, so $\mathrm{u}^{\mathrm{h}}\left(\mathrm{x}_{\mathrm{I}}\right) \neq \mathrm{u}_{\mathrm{I}}$, the nodal parameters $u_{I}$ are not the nodal values of $\mathrm{u}^{\mathrm{h}}\left(\mathrm{x}_{\mathrm{I}}\right)$. Therefore, we use Lagrange multiplier method to enforce the essential boundary conditions.

\section{Choice of Support Domain and Weight Function}

The support domain usually used circular or rectangular. There is no difference if a circular or rectangular support domain is used in the EFG method [4,5]. A weight function needs properties as following:

- Compact support, i.e. zero outside the support domain.

- The values of all points in the support domain is positive.

- The value of its is maximum at the current point and decrease when moving outwards.

There are many kinds of function satisfying for these properties. In this paper, we used the quadratic spline function as follow

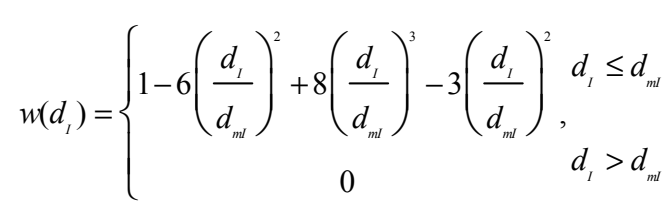

With: $\mathrm{d}_{\mathrm{I}}=\left\|\mathrm{X}-\mathrm{x}_{\mathrm{I}}\right\|$

$\mathrm{d}_{\mathrm{mI}}$ is the radius of influence domain $\mathrm{x}_{\mathrm{I}}$

$$
\mathrm{d}_{\mathrm{mI}}=\mathrm{d}_{\max } \cdot \mathrm{c}_{\mathrm{I}}
$$

Where the scaling parameter $\mathrm{d}_{\max }$ usually is chosen $1.5 \div 4$ for static analysis. The characteristic dimension parameter $c_{I}$ represents the nodal spacing. If the nodes are uniformly distributed then $\mathrm{c}_{\mathrm{I}}$ is the distance between two adjacent nodes.

\section{Discrete Equations and Integration}

In EFGM, the shape functions dissatisfied the Kronecker delta property. Therefore, we have to use Lagrange multiplier to invoke essential boundary. The Lagrange multiplier in [4] as follow as: 


$$
\tilde{L}=L+\int_{s_{u}} \lambda^{T}(u-\bar{u}) d S_{u}
$$

The Lagrange multiplier $(\lambda)$ can be interpreted as the reaction forces needed to fulfill the displacement conditions at the boundary. The approximation given by:

$$
u(x) \cong u^{h}(x)=\sum_{I=1}^{n} \Phi_{I}(x) \cdot u_{I}=\Phi(x) U
$$

By enforcing essential boundary conditions using Lagrange multiplier approach. Discretization of (14) results in [7].

$$
\left[\begin{array}{cc}
K & G \\
G^{T} & o
\end{array}\right]\left\{\begin{array}{l}
U \\
\Lambda
\end{array}\right\}=\left\{\begin{array}{l}
f \\
q
\end{array}\right\}
$$

With $\mathrm{K}$ is stiffness matrix.

$$
K_{I J}=\int_{\Omega} B_{I}^{T} D B_{J} d \Omega
$$

$\mathrm{f}$ is the force vector.

$$
f_{I}=\int_{\Omega} \Phi^{T} b d \Omega+\int_{S_{T}} \Phi^{T} t d S_{T}
$$

$B_{I}$ is the partial derivatives of the shape function.

$$
\begin{gathered}
B_{I}=\left[\begin{array}{cc}
\Phi_{I, x} & 0 \\
0 & \Phi_{I, y} \\
\Phi_{I, y} & \Phi_{I, x}
\end{array}\right] \quad N_{K}=\left[\begin{array}{cc}
N_{K} & 0 \\
0 & N_{K}
\end{array}\right] \\
G_{I K}=\int_{S_{u}}-N^{T} \Phi d S_{u} \quad q_{K}=\int_{S_{u}}-N^{T} \bar{u} d S_{u}
\end{gathered}
$$

For plane stress:

$$
\begin{gathered}
D=\frac{E}{1-v^{2}}\left[\begin{array}{ccc}
1 & v & 0 \\
v & 1 & 0 \\
0 & 0 & (1-v) / 2
\end{array}\right]=D_{e} \\
\sigma=\varepsilon . D
\end{gathered}
$$

\section{The Stress and Displacement near Crack Tip}

The analytical solution for the stresses of an infinite plate $[10,11]$

Mode 1:

$$
\sigma_{x}=\frac{K_{I}}{\sqrt{2 \pi r}} \cos \frac{\theta}{2}\left[1-\sin \frac{\theta}{2} \sin \frac{3 \theta}{2}\right]+O\left(r^{0}\right)
$$

$$
\begin{gathered}
\sigma_{y}=\frac{K_{I}}{\sqrt{2 \pi r}} \cos \frac{\theta}{2}\left[1+\sin \frac{\theta}{2} \sin \frac{3 \theta}{2}\right]+O\left(r^{0}\right) \\
\tau_{x y}=\frac{K_{I}}{\sqrt{2 \pi r}} \sin \frac{\theta}{2} \cos \frac{\theta}{2} \cos \frac{3 \theta}{2}+O\left(r^{0}\right) \\
v=\frac{K_{I}}{4 G} \sqrt{\frac{r}{2 \pi}}\left[(2 k+1) \sin \frac{\theta}{2}-\sin \frac{3 \theta}{2}\right]+O\left(r^{0}\right) \\
u=\frac{K_{I}}{4 G} \sqrt{\frac{r}{2 \pi}}\left[(2 k-1) \cos \frac{\theta}{2}-\cos \frac{3 \theta}{2}\right]+O\left(r^{0}\right)
\end{gathered}
$$

Mode 2:

$$
\begin{aligned}
& \sigma_{x}=-\frac{K_{I I}}{\sqrt{2 \pi r}} \sin \frac{\theta}{2}\left[2+\cos \frac{\theta}{2} \cos \frac{3 \theta}{2}\right]+O\left(r^{0}\right) \\
& \sigma_{y}=\frac{K_{I I}}{\sqrt{2 \pi r}} \sin \frac{\theta}{2} \cos \frac{\theta}{2} \cos \frac{3 \theta}{2}+O\left(r^{0}\right)
\end{aligned}
$$$$
\tau_{x y}=\frac{K_{I I}}{\sqrt{2 \pi r}} \cos \frac{\theta}{2}\left[1-\sin \frac{\theta}{2} \sin \frac{3 \theta}{2}\right]+O\left(r^{0}\right)
$$$$
u=\frac{K_{I I}}{4 G} \sqrt{\frac{r}{2 \pi}}\left[(2 k+3) \sin \frac{\theta}{2}+\sin \frac{3 \theta}{2}\right]+O(r)
$$

$$
v=\frac{K_{I I}}{4 G} \sqrt{\frac{r}{2 \pi}}\left[(2 k-3) \cos \frac{\theta}{2}+\cos \frac{3 \theta}{2}\right]+O(r)
$$$$
w=0
$$

\section{Enrichment Functions}

In the EFG method, by adding extensions in Meshless methods to reflect the discontinuous displacement field generated by the crack. For the case of linear elastic fracture mechanics, two sets of functions are used: a Heaviside jump functions to capture the jump across the crack faces and asymptotic Branch functions that span the 2D asymptotic crack tip fields. The enriched approximation for fracture mechanics problems take the form [12-15]

$$
\begin{aligned}
u^{h}(\boldsymbol{x}) & =\sum_{I \in N} \Phi_{I}(\boldsymbol{x}) u_{I}+\sum_{I \in N^{b}} \Phi_{I}(\boldsymbol{x}) H(\boldsymbol{x}) a_{I} \\
& +\sum_{I \in N^{s}} \Phi_{I}(\boldsymbol{x}) B_{k}^{e n r}(\boldsymbol{x}) \beta_{I}
\end{aligned}
$$

In the equation (26), the first term is the standard approximations functions of EFG methods, the second term reflects discontinuous along the two sides of crack surface, and the third term reflects the crack tip singularity.

Where $\mathrm{N}$ is the entire set of particles in the domain, $\mathrm{N}^{\mathrm{b}}$ is 
the set of particles whose domain of influence is completely bisected by the crack, $\mathrm{N}^{\mathrm{s}}$ is the set of particles whose domain of influence is bisected by the crack tip as shown in Fig.1.

$\mathrm{H}(\mathrm{x})$ is Heaviside jump enriched function, and given by [16]:

$$
H(f(x))=\left\{\begin{array}{ll}
+1 & f(x)>0 \\
-1 & f(x)<0
\end{array}\right\}
$$

$\mathrm{B}(\mathrm{x})$ is Branch enriched function, the crack tip extension function, and given by [17]:

$$
B^{a n}(\mathrm{x})=\left[\begin{array}{lll}
\sqrt{r} \cos \left(\frac{\theta}{2}\right) & \sqrt{r} \sin \left(\frac{\theta}{2}\right) & \sqrt{r} \sin \left(\frac{\theta}{2}\right) \sin \theta \\
\sqrt{r} \cos \left(\frac{\theta}{2}\right) \sin \theta
\end{array}\right]
$$

The Branch enrichment is crucial to accurately locate the crack tip in enriched meshfree methods. The crack tip enrichment ensures that the crack is properly closed at the crack tip.

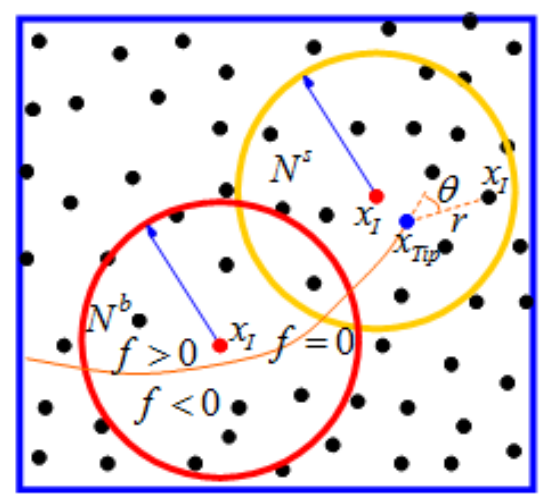

Figure 1. Selection of the support domain near a crack face

Where $f(x)$ is the signed distance function from the crack line; $\mathrm{x}$ is the sample points, $\mathrm{x}_{\mathrm{I}}$ is the distance from the surface cracks to the point $\mathrm{x}$ nearest; $\mathrm{r}$ and $\theta$ are polar coordinates with respect to the crack tip as shown in Fig. 1.

\section{Crack Growth Direction}

The propagation of crack requires a suitable criterion for a crack growth. The commonly used criteria are: the maximum principal stress criterion [18], the maximum energy release rate criterion [19], and the minimum strain energy density criterion [20].

In this study, we use maximum hoop stress theory, which assumes that the crack may grow in a direction perpendicular to the maximum principal stress [21].

$$
\sigma_{r \theta}=\frac{1}{\sqrt{2 \pi r}} \cos \frac{\theta}{2}\left[\frac{1}{2} K_{I} \sin \theta+\frac{1}{2} K_{I I}(3 \cos \theta-1)\right]=0
$$

Where $\mathrm{K}_{\mathrm{I}}$ and $\mathrm{K}_{\mathrm{II}}$ are the stress intensity factor of modes 1 and 2 , respectively. The variables $r$ and $\theta$ are as shown in Fig. 2. Therefore, the crack growth direction $\theta_{0}$ for each crack increment is obtained by following condition [22].

$$
K_{I} \sin \theta_{0}+K_{I I}\left(3 \cos \theta_{0}-1\right)=0
$$

After solving the above equation, we obtain

$$
\theta_{0}=2 \arctan \left[\frac{1}{4}\left(\frac{K_{I}}{K_{I I}}\right) \pm \sqrt{\left(\frac{K_{I}}{K_{I I}}\right)^{2}+8}\right]
$$

According to this criterion, the equivalent mode I SIF is

$$
K_{e}=K_{I} \cos ^{3}\left(\theta_{c} / 2\right)-3 K_{I I} \cos ^{2}\left(\theta_{c} / 2\right) \sin \left(\theta_{c} / 2\right)
$$

When equivalent stress intensity factor $\mathrm{K}_{\mathrm{e}}$ is greater than fracture toughness of the material $\mathrm{K}_{\mathrm{c}}$, cracks began to expand.

$\mathrm{K}_{\mathrm{c}}$ is fracture toughness of the material.

The steps of calculations in Matlab code:

Step 1: Set up the nodal coordinates for a problem domain.

Step 2: Set up a background mesh for numerical integration.

Step 3: Determine the Gauss point, weight function and Jacôbi.

Step 4: Determine the domain of influence of each node in the model.

Step 5: Determine shape functions MLS and shape function derivatives.

Step 6: Enriched.

Step 7: Determine stiffness matrix K.

Step 8: Enforce essential boundary conditions using Lagrange multipliers.

Step 9: Assembled to form the master stiffness matrix and solve for equations.

Step 10: Solve for nodal parameters, solve for stresses.

Step 11: Solve for stress intensity factors and direction of crack propagation.

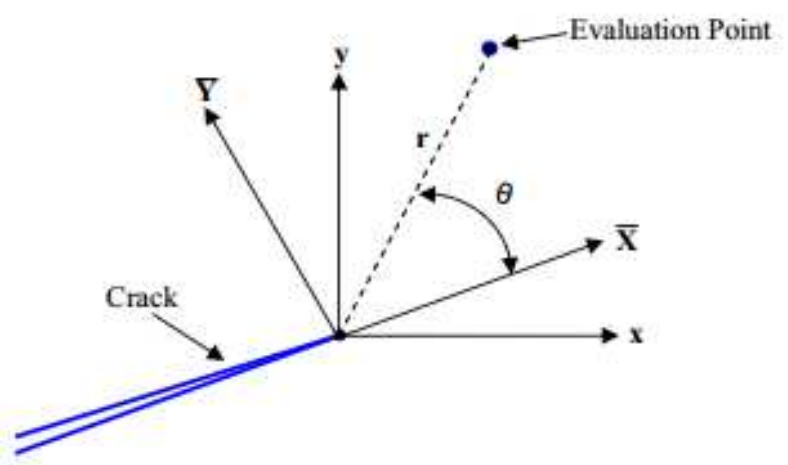

Figure 2. Distance $r$ and angle $\theta$ of a point $\boldsymbol{x}$ the crack tip

\section{Numerical Simulation}

\subsection{Rectangular Plate with a Center Crack under Tension Shown in Fig. 3.}

The plate has an initial crack length of $2 a=40 \mathrm{~cm}$, a plate length of $\mathrm{H}=350 \mathrm{~cm}$, a plate width of $b=175 \mathrm{~cm}$; Elastic modulus $E=2 \times 10^{7} \mathrm{~N} / \mathrm{cm}^{2}$; Poisson's ratio $v=0.3$. Fracture 
toughness $K_{c}=140 \mathrm{~N} / \mathrm{cm}^{3 / 2}$; The dimensionless size of support domain $\mathrm{d}_{\max }=1.75$; a $4 \times 4$ Gauss quadrature is used and $6 \times 6$ nodes around the crack tip are selected for enrichment.
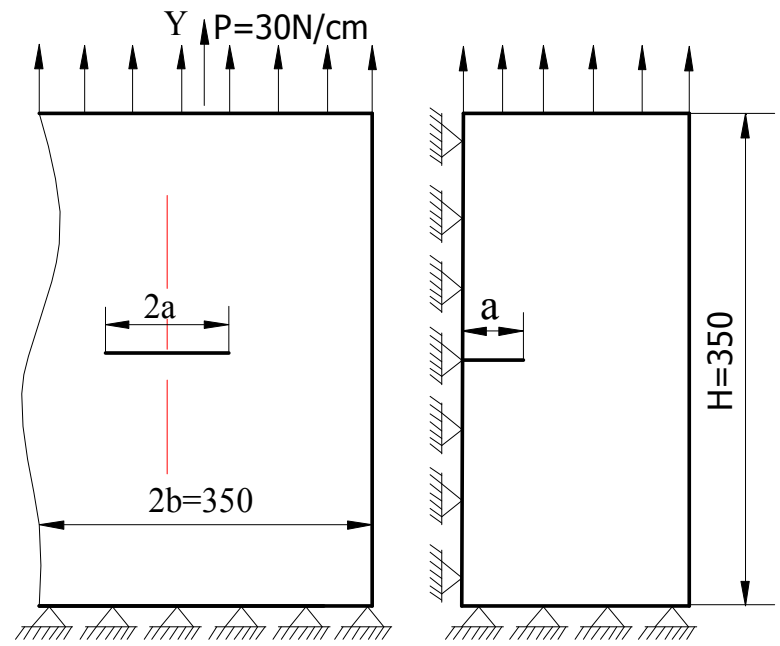

Figure 3. Mode I crack subjected to tensile load

Thanks to the symmetry, only half of the model calculations.

The reference mode I SIF is given by [11]

$$
K_{\text {Theory }}=\sigma \sqrt{\pi a F_{1}}(a / b)
$$

Where $a$ is the crack length, $b$ is the plate width and $F_{I}(a / b)$ is an empirical function given as

$$
\begin{gathered}
F_{1}(a / b)=\left[1-0.025(a / b)^{2}+0.06(a / b)^{4}\right] \sqrt{\sec \frac{\pi a}{2 b}} \\
\operatorname{Error}(\%)=\frac{K_{E F G}-K_{F E M}}{K_{E F G}} .100 \%
\end{gathered}
$$

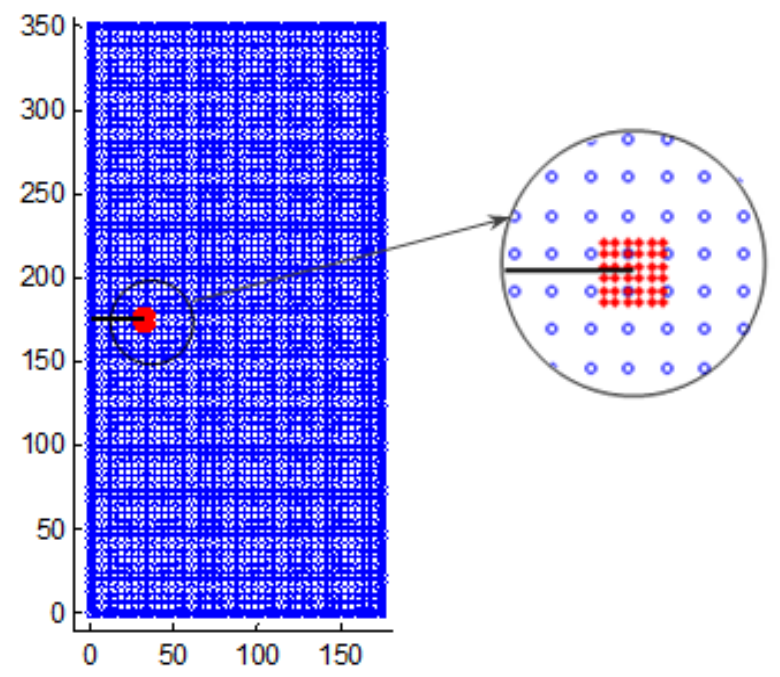

Figure 4. Nodal discretization and around the crack tips was refined with $6 x 6$ nodes

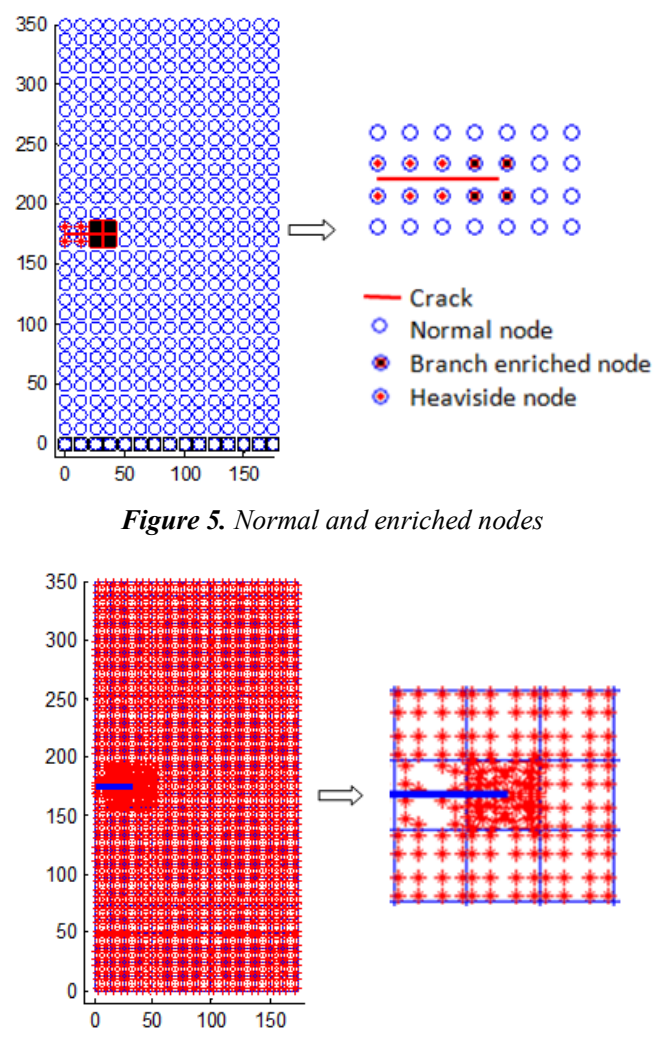

Figure 6. Enriched nodes and Gauss point distribution $4 x 4$

\subsubsection{Analysis of the Number of Nodes in the SIF Calculation}

Table 1. Shows the mode I SIFs obtained using different of the number of nodes

\begin{tabular}{llll}
\hline Nodes & $\mathbf{K}_{\mathbf{E F G}}$ & $\mathbf{K}_{\text {Theory }}$ & $\mathbf{K}_{\text {FEM }}[\mathbf{2 3}]$ \\
\hline $10 \times 20$ & 247,2613 & 239,6579 & 255,9088 \\
$15 \times 30$ & 242,3825 & 239,6579 & 252,3577 \\
$20 \times 40$ & 241,0894 & 239,6579 & 245,3904 \\
$25 \times 50$ & 241,116 & 239,6579 & 243,7217 \\
$30 \times 60$ & 241,2555 & 239,6579 & 243,5303 \\
$35 \times 70$ & 241,1846 & 239,6579 & 243,511 \\
$40 \times 80$ & 241,0183 & 239,6579 & 243,6795 \\
$45 \times 90$ & 241,2739 & 239,6579 & 243,9402 \\
\hline
\end{tabular}

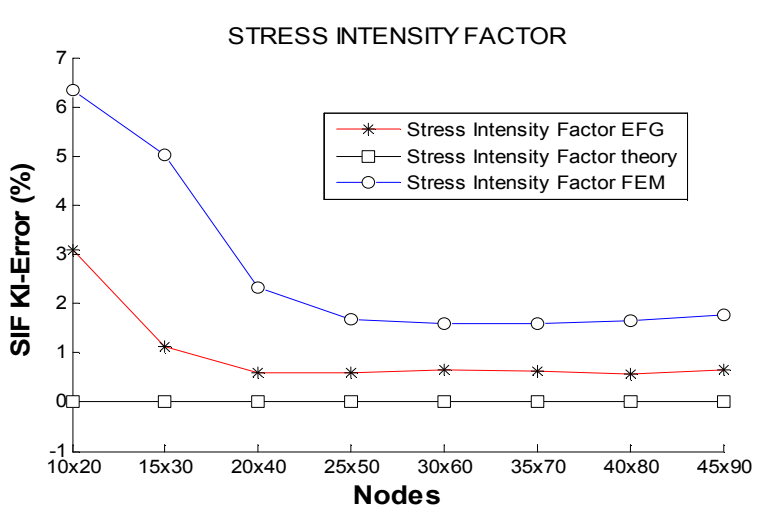

Figure 7. SIF \% error of different nodes density

The results obtained in Table 1 show that the accuracy of the EFG method depend on the smoothing node density. If 
the node density is as smoothing as a high precision and vice versa. This article demonstrates that the problem of distributed nodes in EFG method great influence to the accuracy of calculations. To increase the accuracy level, we just simply add the button to the crack tip. Because no grid structure, hence the problem of adding nodes can be implemented and reduce computation time.

Using the results of EFG method compared to the results of element finite method and exact method, the values of error are mostly less than $1 \%$ is shown in Fig. 7.

\subsubsection{Analysis of the Crack Length in the SIF Calculation. With $a=i * 5(\mathrm{~cm})(i=1: 10)$}

Table 2. shows the mode I SIFs obtained using different of the crack length

\begin{tabular}{lllll}
\hline $\boldsymbol{a}(\boldsymbol{c m})$ & $\boldsymbol{N e l}$ & $\boldsymbol{K}_{\boldsymbol{E F G}}$ & $\boldsymbol{K}_{\text {theory }}$ & $\boldsymbol{K}_{\boldsymbol{F E M}}[\mathbf{2 3}]$ \\
\hline 5 & $40 \times 80$ & 119.5638 & 118.9573 & 118,09 \\
10 & $40 \times 80$ & 169.3015 & 168.4756 & 167.1297 \\
15 & $40 \times 80$ & 207.8535 & 206.8415 & 203.7212 \\
20 & $40 \times 80$ & 241.0183 & 239.6579 & 237.8907 \\
25 & $40 \times 80$ & 270.8392 & 269.1344 & 267.5569 \\
30 & $40 \times 80$ & 298.436 & 296.4354 & 295.4391 \\
35 & $40 \times 80$ & 324.9144 & 322.2807 & 322.2666 \\
40 & $40 \times 80$ & 350.4446 & 347.1662 & 348.433 \\
45 & $40 \times 80$ & 375.588 & 371.463 & 374.166 \\
50 & $40 \times 80$ & 399.6855 & 395.4695 & 399.5562 \\
\hline
\end{tabular}

From table 2, we can show that the different of the crack length under three different approaches. In Fig. 8 we also see that error of EFG method is small. When the crack length a = $45 \mathrm{~cm}$, the maximum error is about $1.0982 \%$, the crack length $\mathrm{a}=15 \mathrm{~cm}$, the minimum error of approximately $0.4868 \%$.

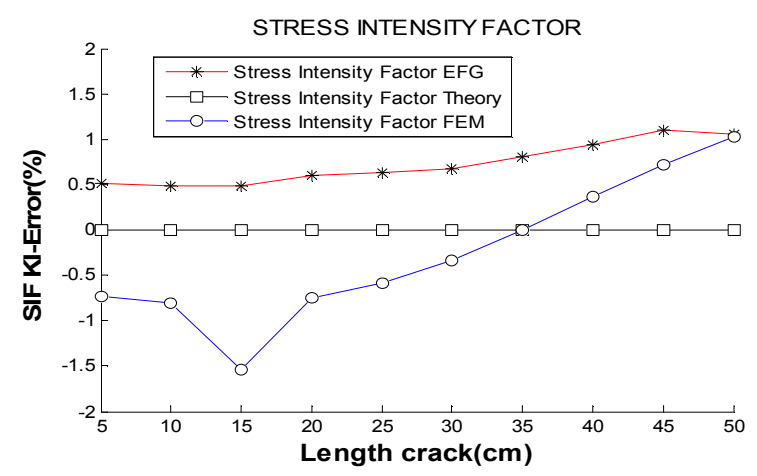

Figure 8. SIF\% error of different crack length

\subsubsection{Analysis Dimensionless Size of the Support Domain ( $\left.d_{\text {max }}\right)$ in the SIF Calculation}

Table 3. shows the mode I SIFs obtained using different of $d_{\max }$

\begin{tabular}{ccccc}
\hline $\boldsymbol{d}_{\boldsymbol{m a x}}$ & $\boldsymbol{N e \boldsymbol { l }}$ & $\boldsymbol{K}_{\boldsymbol{E F G}}$ & $\boldsymbol{K}_{\text {Theory }}$ & Error (\%) \\
\hline 1.6 & $20 \times 40$ & 297,1386 & 306,9168 & $-3,2907$ \\
1.7 & $20 \times 40$ & 300,1515 & 306,9168 & $-2,2539$ \\
1.8 & $20 \times 40$ & 298,9854 & 306,9168 & $-2,6527$ \\
1.9 & $20 \times 40$ & 297,4066 & 306,9168 & $-3,1977$ \\
2.0 & $20 \times 40$ & 299,0257 & 306,9168 & $-2,6389$ \\
2.1 & $20 \times 40$ & 301,3948 & 306,9168 & $-1,8321$ \\
2.2 & $20 \times 40$ & 306,14 & 306,9168 & $-0,2537$ \\
2.3 & $20 \times 40$ & 317,113 & 306,9168 & 3,2153 \\
\hline
\end{tabular}

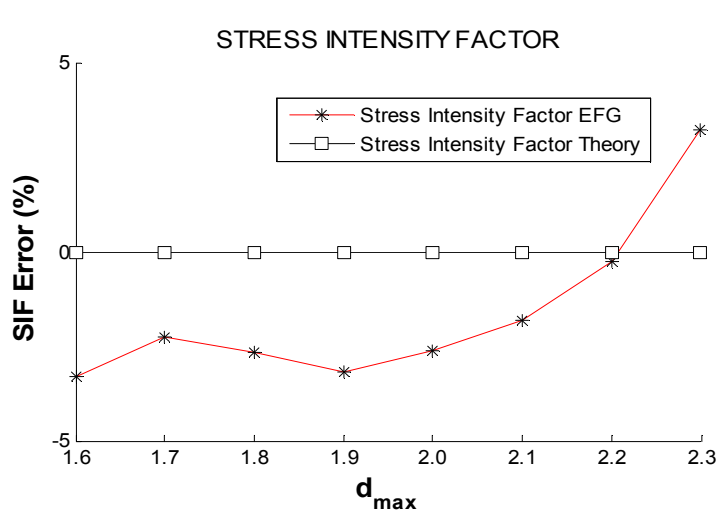

Figure 9. SIF\% error of different $d_{\max }$

Table 3 shows that the size of nodes in the influence regions have an important role in Meshless methods because the results obtained of Meshless method based on influence domain by using moving least squares to solve point within the variable. If the domain of influence parameters are chosen as $\mathrm{d}_{\max }=1.7 \div 2.2$ then computational precision is high. Conversely, $d_{\max }$ is chosen outside this region then the effectiveness of approximation is not good.

\subsubsection{Analysis of the Tension in the SIF Calculation: $q=5 * i$ $(\mathrm{N} / \mathrm{cm})(i=1: 10)$}

From Fig. 10, we find that the calculation error is independent of the distribution of the load q. All computational errors always equal $0.8280 \%$.

Table 4. shows the mode I SIFs obtained using different of load

\begin{tabular}{lllll}
\hline $\boldsymbol{q}(\boldsymbol{N} / \mathrm{cm})$ & $\boldsymbol{N e l}$ & $\boldsymbol{K}_{\boldsymbol{E F G}}$ & $\boldsymbol{K}_{\text {Theory }}$ & $\boldsymbol{K}_{\text {FEM }}[\mathbf{2 3}]$ \\
\hline 5 & $20 \times 40$ & 40,2263 & 39,9429 & 39,3728 \\
10 & $20 \times 40$ & 80,4525 & 79,8859 & 78,745792 \\
15 & $20 \times 40$ & 120,6788 & 119,8289 & 118,1186 \\
20 & $20 \times 40$ & 160,9051 & 159,7719 & 157,4915 \\
25 & $20 \times 40$ & 201,1314 & 199,7149 & 196,86448 \\
30 & $20 \times 40$ & 241,3576 & 239,6579 & 236,2373 \\
35 & $20 \times 40$ & 281,5839 & 279,6009 & 275,61027 \\
40 & $20 \times 40$ & 321,8102 & 319,5438 & 314,9831 \\
45 & $20 \times 40$ & 362,0365 & 359,4868 & 354,3561 \\
50 & $20 \times 40$ & 402,2627 & 399,4298 & 393,7289 \\
\hline
\end{tabular}

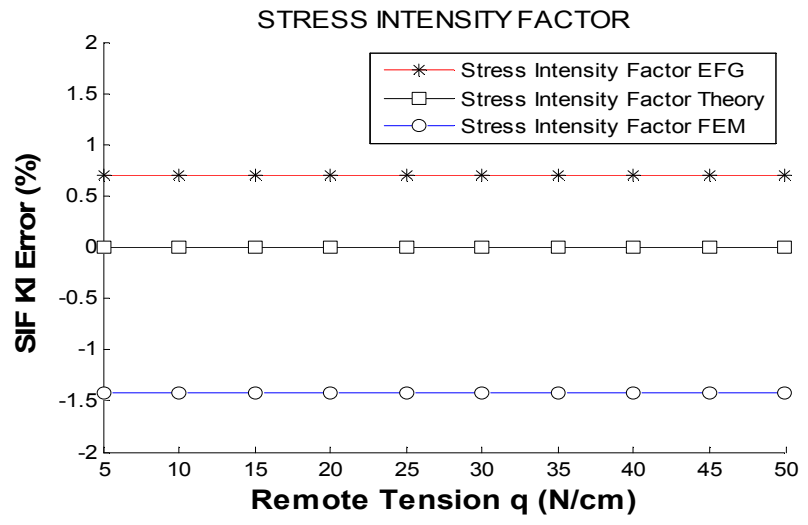

Figure 10. SIF \% error of different load 


\subsection{Rectangular Plate with a Single Edge Crack under Tension Shown in Fig. 5.}

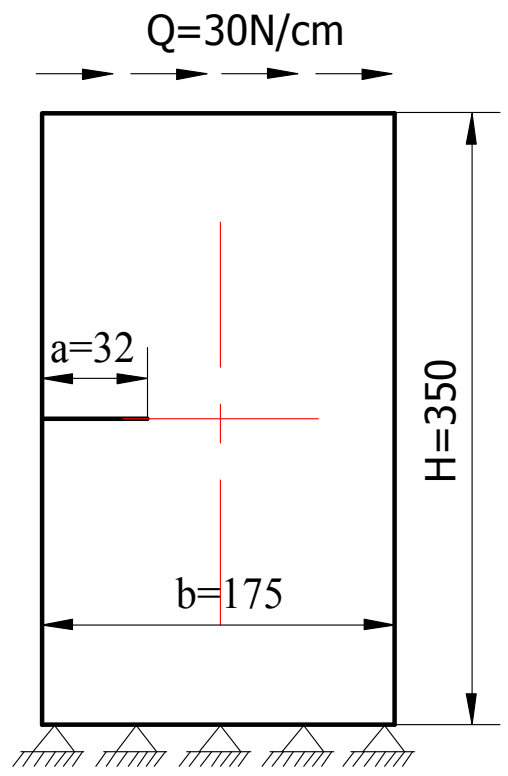

Figure 11. Single Edge Crack Computational model (mode2)

The plate has an initial crack length of $a=32 \mathrm{~cm}$, a plate length of $\mathrm{H}=350 \mathrm{~cm}$, a plate width of $b=175 \mathrm{~cm}$; Elastic modulus $E=2 \times 10^{7} \mathrm{~N} / \mathrm{cm}^{2}$; Poisson's ratio $v=0.3$. Fracture toughness: $K_{c}=140 \mathrm{~N} / \mathrm{cm}^{3 / 2}$; The dimensionless size of support domain $\mathrm{d}_{\max }=1.75$;

A Gauss integration of $4 \times 4$ orders is used, without local refinement at the crack tip.

The reference mode II SIF is given by [11]

$$
K_{\text {II heory }}=\sigma \sqrt{\pi a} \cdot F_{I I}\left(\frac{a}{b}\right)
$$

Where $a$ is the crack length, $b$ is the plate width and $F_{I I}(a / b)$ is an empirical function given as

$$
\begin{aligned}
F_{I I}\left(\frac{a}{b}\right)= & 1.122-0.231\left(\frac{a}{b}\right)+10.55\left(\frac{a}{b}\right)^{2} \\
& -21.718\left(\frac{a}{b}\right)^{3}+30.382\left(\frac{a}{b}\right)^{4}
\end{aligned}
$$

\subsubsection{Analysis of the Number of Nodes in the SIF $K_{I I}$ Calculation}

Table 5. Analysis different of the number of nodes in the SIF calculation

\begin{tabular}{llll}
\hline Nel & $\boldsymbol{K}_{\boldsymbol{I I}_{\boldsymbol{E F G}}}$ & $\mathbf{K}_{\mathbf{I I}}{ }_{\text {Theory }}$ & $\boldsymbol{K}_{\boldsymbol{I I}}[\mathbf{F E M}$ \\
\hline $30 \times 60$ & 395,5081 & 401,1702 & 404,2531 \\
$35 \times 70$ & 396,2977 & 401,1702 & 403,3656 \\
$40 \times 80$ & 397,0112 & 401,1702 & 400,2353 \\
$45 \times 90$ & 397,8484 & 401,1702 & 399,7724 \\
$50 \times 100$ & 397,879 & 401,1702 & 399,4941 \\
$55 \times 110$ & 397,8911 & 401,1702 & 399,3723 \\
$60 \times 120$ & 397,9574 & 401,1702 & 399,3572 \\
$65 \times 130$ & 398,0053 & 401,1702 & 399,3854 \\
\hline
\end{tabular}

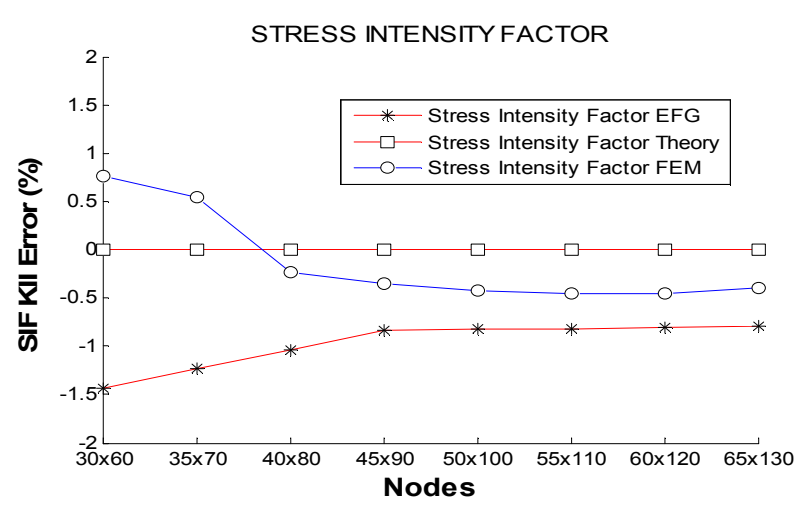

Figure 12. SIF $K_{I I} \%$ error of different nodes density

In this section, computational analysis without smoothing at the crack tip.

The results obtained of Table 5 illustrates that the maximum error of EFG method and element finite method with the same number of nodes is $1.40 \%$ and $0.76 \%$, respectively. When the mesh density is smoothing then computational precision is high. Thus, we can find that the computational precision depends greatly on the distribution of nodes.

\subsubsection{Analysis of the Crack Length in the SIF $K_{I I}$ Calculation}

Table 6. Analysis of the crack length in the SIF calculation. With $a=i * 10(\mathrm{~cm})$ $(i=1: 8)$

\begin{tabular}{lllll}
\hline $\mathbf{a}(\mathbf{c m})$ & $\boldsymbol{N e l}$ & $\boldsymbol{K}_{\boldsymbol{I I F G}}$ & $\mathbf{K}_{\text {II }}$ & $\boldsymbol{K}_{\boldsymbol{I I}}$ [23] \\
\hline 10 & $65 \times 130$ & 190,7816 & 191,6101 & 194,8391 \\
20 & $65 \times 130$ & 285,7325 & 286,8245 & 290,5711 \\
30 & $65 \times 130$ & 378,9788 & 381,316 & 384,4031 \\
40 & $65 \times 130$ & 482,2136 & 485,6039 & 487,7887 \\
50 & $65 \times 130$ & 602,4452 & 606,5331 & 609,3197 \\
60 & $65 \times 130$ & 750,6046 & 752,7061 & 757,2961 \\
70 & $65 \times 130$ & 933,2459 & 936,6675 & 942,2688 \\
80 & $65 \times 130$ & 1172,198 & 1176,25 & 1179,348 \\
\hline
\end{tabular}

From Table 6 and Fig. 3 show that the error of EFG method are small. When the crack length $\mathrm{a}=40 \mathrm{~cm}$, the maximum error calculation is $0.7030 \%$. When the crack length $\mathrm{a}=60 \mathrm{~cm}$, the minimum error calculation is $0.2799 \%$.

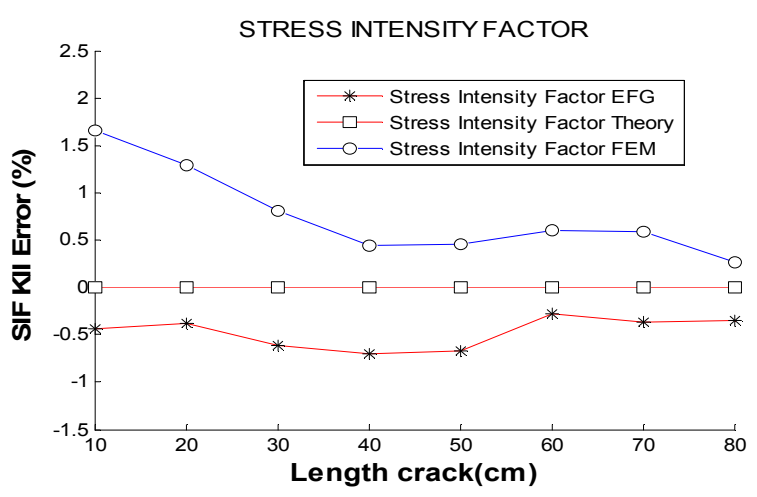

Figure 13. SIF KII \% error of different crack length 


\subsection{Simulation Results of Displacement Fields and Stress Field}

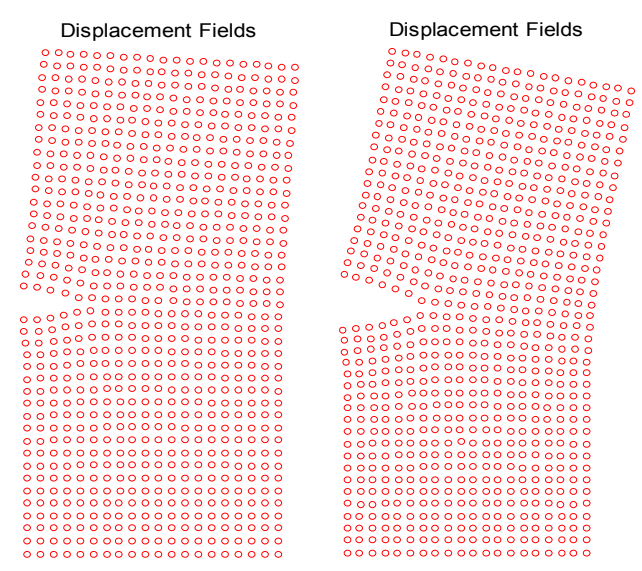

Figure 14. The node displacement fields after 2 steps

Fig. 14 presents the displacement field of edge crack finite plates. Where we can see that the node displacement fields after 2 steps is reflected under the effect of loads.

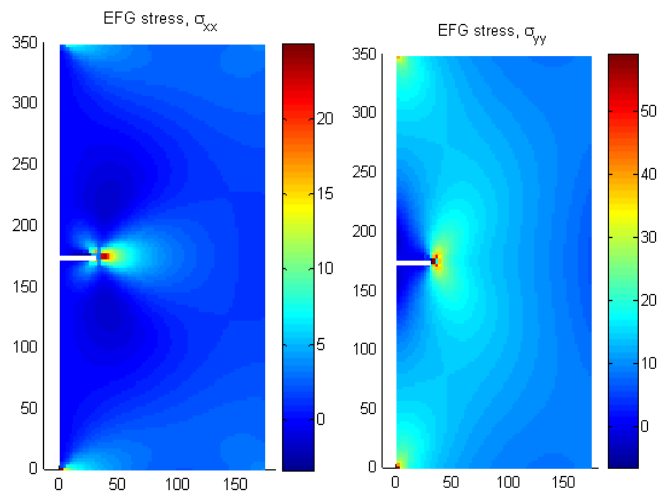

Figure 15. $x$, $y$ direction stress field of center crack under tension

Fig. 15 and Fig. 16 present the stress contour plot of $\sigma_{x x}$ and $\sigma_{y y}$ direction of center and edge crack under tension. It also shows that the stress concentration at the crack, the singularity of the crack tip stress and and the stress field at the crack location outside is smooth.

All the problems have been simulated by EFG codes (algorithms) writting in MATLAB (R2010b).

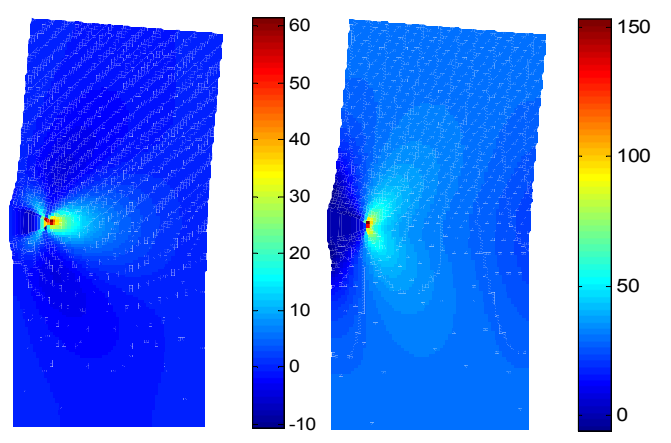

Figure 16. $x$, $y$ direction stress field of single edge crack under tension

\subsection{Simulation of Crack Propagation}

Simulate the growth of crack in quasi-static is evaluated by equivalent stress intensity factor, and if its exceeds the fracture toughness of the material then the crack will be extended for some finite length (da) in a particular direction that is found by a suitable crack growth criterion. The step size (da) is a used to determine parameters, and da should be chosen enough minor to get an accurate crack growth path. The stress intensity factors are recalculated for the new crack geometry, and the next crack is extended according to a new direction.

In this paper, the following equation is calculated for each step of the cracking step [24]:

$$
d a_{n}=\frac{K_{e}^{(n)}}{K_{e}^{(0)}} \cdot d a_{0}
$$

Where $\mathrm{da}_{0}$ is initial cracking step, $K_{e}^{(0)}$ is initial equivalent stress intensity factor, $\mathrm{da}_{\mathrm{n}}$ is step $\mathrm{n}$ for cracking step, $K_{e}^{(\mathrm{n})}$ is step $\mathrm{n}$ the equivalent stress intensity factor.

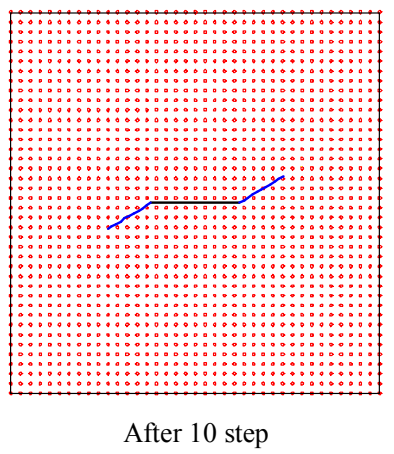

Figure 17. Crack growth path of centre crack

The obtained results of model crack propagation analysis by using a relatively coarse discretization of $40 \times 40$ nodes for centre crack and 20x40 nodes for edge crack. The crack growth increment is selected $0.1 \mathrm{a}(\mathrm{a}=40)$ for this study, and the crack growth are simulated for 10 steps and 15 step. The result of crack path is shown as Fig. 17 and Fig. 18.

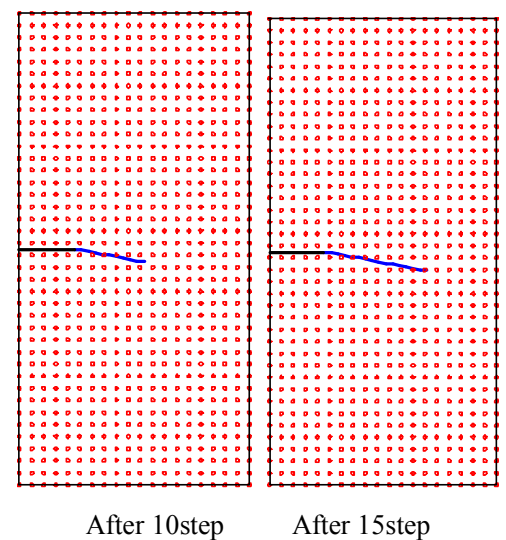

Figure 18. Crack growth path of single edge crack 


\section{Conclusions and Discussion}

The Meshless computation has some outstanding advantages compare to the traditional finite element method (FEM) in treatment of arbitrary evolving discontinuity. Because of the independence of elements, the adaptive refinement can be easily achieved. Therefore, the crackpropagation analysis can be done easily and dramatically simplified. By introducing enriched functions, the extensions are added in the approximation of traditional Meshless method, the computation accuracy was improved. Through the computational results of stress intensity factors for edge and centre crack are compared to the results of element finite method and exact method, proving the Meshless method is very convenient for crack problem. In both cases the crack growth that is edge and centre crack are simulated for predicting fatigue crack propagation path. This method is used to allow crack growth without remeshing.

Through the analyses of numerical examples demonstrate, we can see that enriched EFG method which can solve the fracture problems is effective, and has practical merits for modeling crack growth problem. It is very promising in engineering application.

\section{References}

[1] S.T. Raveendra, P.K. Banerjee, "Boundary element analysis of cracks in thermally stresses planar structures," Int. J. Solids Struct, vol. 29, 1992, pp. 2301-2317.

[2] L.N. Gifford, P.D. Hilton, Stress intensity factors by enriched finite elements, Eng. Fract. Mech, vol.10, 1978, pp. 485-496.

[3] M. Duflot, "The extended finite element method in thermoelastic fracture mechanics," Int. J. Numer. Methods Eng, Vol.74, 2008, pp. 827-847.

[4] Belytschko T, Lu YY, Gu L, "Element-free Galerkin methods," International Journal for Numerical Methods in Engineering, 1994, vol.37, pp. 229-256.

[5] Fleming M, Chu YA, Moran B, Belytschko T, "Enriched element-free Galerkin methods for crack tip fields," Int J Numer Methods Eng, 1997, vol.40, pp. 1483-504.

[6] Belytschko T, Gu L, Lu YY, "Fracture and crack growth by element-free Galerkin methods," Modelling and Simulation in Materials Science and Engineering, 1994, vol. 12, pp. 519-534

[7] Belytschko T, Lu YY, Gu L, Tabbara M, "Element-free Galerkin methods for static and dynamic fracture," International Journal of Solids and Structures 1995, vol.32, pp. $2547-2570$

[8] Belytschko T, Tabbara M, "Dynamic fracture using elementfree Galerkin methods," International Journal for Numerical Methods in Engineering, 1996, vol.39, pp. 923-938.

[9] Lancaster P, Salkauskas K, "Surfaces generated by moving least squares methods," Math Comput, 1981, vol. 37, pp. 14158 .
[10] Nguyen P, Rabczuk T, Bordas S, Duflot M, "Meshless methods: a review and Computer implementation aspects," Math Comput Simul, 2008, vol.79, pp. 763-813.

[11] Tada Hiroshi, Paris Paul, Irwin George, “The Stress Analysis of Cracks Handbook [M]," Washington University, 1957.

[12] Dennis M Tracy, "Finite elements for determination of crack tip elastic stress intensity factors [J]," Engineering Fracture Mechanics, 1971, vol.3(3), pp. 255-266.

[13] Moes N, Dolbow J, Belytschko T, "A finite element method for crack growth without remeshing," International Journal for Numerical Methods in Engineering, 1999, vol.46(1), pp.131150 .

[14] Ma wen tao, Li ning, Shi jun ping, "Modelling crack growth by enriched Meshless method based on partition of unity," Chinese journal of computational Mechanics, 2013, vol.30, pp.28-33.

[15] N. Muthu, S. K. Maiti, B. G. Falzon, I. Guiamatsia, "A comparison of stress intensity factors obtained through crack closure integral and other approaches using Xtended elementfree Galerkin method," Comput Mech, 2013, vol.52, pp. 587605 .

[16] N. Muthu, B.G.Falzon, S.K.Maiti, S.Khoddam, "Modified crack closure integral technique for extraction of SIFs in mesh free methods," Finite Elements in Analysis and Design, 2014, pp. 25-39.

[17] Sayyed Shahram Ghorashi, Soheil Mohammadi, Saeed-Reza Sabbagh-Yazdi, "Orthotropic enriched element free Galerkin method for fracture analysis of composites" Engineering Fracture Mechanics, vol.78, 2012, pp. 1906-1927.

[18] Kong, X.M., Schluter, N. Dahl, W, "Effect of triaxial stress on mixed-mode fracture," Eng. Fract. Mech, 1995, vol.52(2), pp. 379-388.

[19] Khan, Sh.M.A, Khraisheh. M.K, "A new criterion for mixed mode fracture initiation based on the crack tip plastic core region," Int. J. Plast, 2004, vol.1, pp. 55-84.

[20] Himanshu Pathak, Akhilendra Singh, Indra Vir Singh, "Fatigue crack growth simulations of homogeneous and bimaterial interfacial cracks using element free Galerkin method," Applied Mathematical Modelling, 2013, pp. 11-30.

[21] Erdogan F, Sih GC, "On the crack extension in plates under plane loading and transverse shear," J Basic Eng, 1963, vol.85, pp. 19-27.

[22] I.V. Singh, B.K. Mishra, S. Bhattacharya, R.U. Patil, "The numerical simulation of fatigue crack growth using extended finite element method," Int. J. Fatigue, 2011, vol.36, pp. 109119.

[23] BuiManhTuan, Chenyunfei, "Determine stress intensity factors and stress distribution for a surface crack in plates" Journal of Southeast University, 2014, vol.44 (4), pp. 728-734.

[24] Marc Duflot, Hung Nguyen-Dang, "A meshless method with enriched weight functions for fatigue crack growth," Int. J. Numer. Meth. Engng, 2004, vol.59, pp. 1945-1961. 